PROCEEDINGS OF THE

AMERICAN MATHEMATICAL SOCIETY

Volume 130, Number 6, Pages 1619-1622

S 0002-9939(01)06303-1

Article electronically published on November 15, 2001

\title{
A THEOREM ON THE $k$-ADIC REPRESENTATION OF POSITIVE INTEGERS
}

\author{
YUGUANG FANG
}

(Communicated by David E. Rohrlich)

\begin{abstract}
In this paper, a theorem on the asymptotic property of a summation of digits in a $k$-adic representation is presented.
\end{abstract}

Let $k>1$ be a fixed integer. Then any positive integer $x$ can be uniquely represented by the following $k$-adic form:

$$
x=a_{1} k^{n_{1}}+a_{2} k^{n_{2}}+\cdots+a_{t} k^{n_{t}},
$$

where $n_{1}>n_{2}>\cdots>n_{t} \geq 0$ are integers and $a_{1}, a_{2}, \ldots, a_{t}$ are nonnegative integers not exceeding $k-1$. Define

$$
\alpha(x)=\sum_{i=1}^{t} a_{i}, \quad A(x)=\sum_{y \leq x} \alpha(y) .
$$

In 1940, Bush ([1]) showed that

$$
A(x)=\frac{k-1}{2 \log k} x \log x+o(x \log x),
$$

where log denotes the natural logarithm. In 1948, Bellman and Shapiro ([2]) improved this result and proved that

$$
A(x)=\frac{k-1}{2 \log k} x \log x+O(x \log \log x)
$$

for $k=2$. In 1949, Mirsky ([3]) showed that the $O$-term can be replaced by $O(x)$ for any $k \geq 2$. In 1955, Cheo and Yien (4 $)$ gave another proof for the result and obtained:

$$
A(x)=\frac{k-1}{2 \log k} x \log x+O(x),
$$

Received by the editors January 10, 2001.

2000 Mathematics Subject Classification. Primary 11A63, 11A25, 11 N37.

Key words and phrases. $k$-adic, asymptotic property, arithmetic function, number theory.

(C)2001 American Mathematical Society 
and proved that $O(x)$ cannot be replaced by $O\left(x^{t}\right)$ for any fixed $t<1$. Their proof relies on the identity

$$
\begin{aligned}
A(x)= & \frac{n_{1}(k-1)}{2} \sum_{i=1}^{t} a_{i} k^{n_{i}}-\frac{k-1}{2} \sum_{i=1}^{t}\left(n_{1}-n_{i}\right) a_{i} k^{n_{i}} \\
& +\frac{1}{2} \sum_{i=1}^{t} a_{i}\left(a_{i}-1\right) k^{n_{i}}+\sum_{i=1}^{t} a_{i}+\sum_{i=1}^{t}\left(\sum_{j=1}^{i-1} a_{j}\right) a_{i} k^{n_{i}},
\end{aligned}
$$

where $a_{i}, n_{i}$, and $t$ are as in (11). The first sum equals $\frac{1}{2}(k-1)[\log x / \log k] x$ and the four other sums are shown to be $O(x)$ after complicated mathematical manipulations.

In this paper, we apply a different identity and obtain an estimate on the constant contained in $O(x)$, consequently providing a much simpler proof to the previously known results. The following result is obtained.

Theorem. For any integer $k \geq 2$, we have

$$
A(x)=\frac{k-1}{2 \log k} x \log x+\theta(x) x,
$$

where

$$
-\frac{5 k-4}{8} \leq \theta(x) \leq \frac{k+1}{2} .
$$

To prove this Theorem, we need the following result due to J. L. Lagrange:

Lemma ([5]).

$$
\frac{n-\alpha(n)}{k-1}=\sum_{r=1}^{\infty}\left[\frac{n}{k^{r}}\right],
$$

where $[a]$ denotes the integral part of the real number $a$.

Proof of the Theorem. Using the Lemma, we have

$$
\begin{aligned}
A(x)= & \sum_{n \leq x}\left(n-(k-1) \sum_{r=1}^{\infty}\left[\frac{n}{k^{r}}\right]\right) \\
= & \frac{1}{2} x(x+1)-(k-1) \sum_{r=1}^{\infty} \sum_{n \leq x}\left[\frac{n}{k^{r}}\right] \\
= & \frac{1}{2} x(x+1)-(k-1) \sum_{1 \leq r \leq \log _{k} x}\left(\frac{1}{2}\left[\frac{x}{k^{r}}\right]\left(\left[\frac{x}{k^{r}}\right]-1\right) k^{r}\right. \\
= & \left.\frac{1}{2} x(x+1)+\frac{1}{2}(k-1) \sum_{1 \leq r \leq \log _{k} x} k^{r}\left[\frac{x}{k^{r}}\right]-\left(x-\left[\frac{x}{k^{r}}\right] k^{r}+1\right)\right) \\
& -(k-1) \sum_{1 \leq r \leq \log _{k} x}\left(x\left[\frac{x}{k^{r}}\right]-\frac{1}{2}\left[\frac{x}{k^{r}}\right]^{2} k^{r}\right) .
\end{aligned}
$$


However, we observe that

$$
\begin{gathered}
\sum_{1 \leq r \leq \log _{k} x} k^{r}\left[\frac{x}{k^{r}}\right]=x\left[\log _{k} x\right]+\sum_{1 \leq r \leq \log _{k} x} k^{r}\left(\left[\frac{x}{k^{r}}\right]-\frac{x}{k^{r}}\right) \\
=x \log _{k} x-\theta_{1}(x) x+\sum_{1 \leq r \leq \log _{k} x} k^{r}\left(\left[\frac{x}{k^{r}}\right]-\frac{x}{k^{r}}\right), \\
\sum_{1 \leq r \leq \log _{k} x}\left(x\left[\frac{x}{k^{r}}\right]-\frac{1}{2}\left[\frac{x}{k^{r}}\right]^{2} k^{r}\right)=\frac{1}{2} \sum_{1 \leq r \leq \log _{k} x}\left(\frac{x^{2}}{k^{r}}-k^{r}\left(\left[\frac{x}{k^{r}}\right]-\frac{x}{k^{r}}\right)^{2}\right) \\
=\frac{1}{2} x^{2} \sum_{1 \leq r \leq \log _{k} x} \frac{1}{k^{r}}-\frac{1}{2} \sum_{1 \leq r \leq \log _{k} x} k^{r}\left(\left[\frac{x}{k^{r}}\right]-\frac{x}{k^{r}}\right)^{2},
\end{gathered}
$$

where $0 \leq \theta_{1}(x)<1$. Taking these into (8), we obtain

$$
\begin{aligned}
A(x)= & \frac{1}{2} x(x+1)+\frac{k-1}{2} x \log _{k} x-\frac{k-1}{2} \theta_{1}(x) x-(k-1) \sum_{1 \leq r \leq \log _{k} x}\left[\frac{x}{k^{r}}\right] \\
& -\frac{1}{2} \sum_{1 \leq r \leq \log _{k} x}\left(\left\{\frac{x}{k^{r}}\right\}-\left\{\frac{x}{k^{r}}\right\}^{2}\right) k^{r}-\frac{k-1}{2} x^{2} \sum_{1 \leq r \leq \log _{k} x} \frac{1}{k^{r}},
\end{aligned}
$$

where $\{a\}$ denotes the fractional part of the real number $a$. Using the following inequalities $0 \leq x-x^{2} \leq 1 / 4(0 \leq x \leq 1)$ and $[a] \leq a$, we can find $0 \leq \theta_{2}(x) \leq 1$ and $0 \leq \theta_{3}(x) \leq 1$ such that

$$
\begin{gathered}
\sum_{1 \leq r \leq \log _{k} x}\left[\frac{x}{k^{r}}\right]=\theta_{2}(x) \frac{x}{k-1}, \\
\sum_{1 \leq r \leq \log _{k} x}\left(\left\{\frac{x}{k^{r}}\right\}-\left\{\frac{x}{k^{r}}\right\}^{2}\right) k^{r}=\theta_{3}(x) \frac{k x}{4(k-1)}, \\
x^{2} \sum_{1 \leq r \leq \log _{k} x} \frac{1}{k^{r}}=\frac{x^{2}}{k-1}-\frac{1}{k-1} \frac{x^{2}}{k^{\left[\log _{k} x\right]}} .
\end{gathered}
$$

Substituting these into (9), we finally arrive at

$$
\begin{aligned}
A(x) & =\frac{k-1}{2} \frac{x \log x}{\log k}+\left(-\frac{k-1}{2} \theta_{1}(x)-\theta_{2}(x)+\frac{1}{2}-\frac{k}{8} \theta_{3}(x)+\frac{x}{2 k^{\left[\log _{k} x\right]}}\right) x \\
& =\frac{k-1}{2} \frac{x \log x}{\log k}+\theta(x) x
\end{aligned}
$$

where

$$
-\frac{5 k-4}{8} \leq \theta(x) \leq \frac{k+1}{2}
$$

This completes the proof.

\section{REFERENCES}

1. L. E. Bush, An asymptotic formula for the average sum of the digits of integers, Amer. Math. Monthly 47 (1940), 154-156. MR 1:199f

2. R. Bellman and H. N. Shapiro, On a problem in additive number theory, Ann. of Math. (2) 49 (1948), 333-340. MR 9:414a

3. L. Mirsky, A theorem on representation of integers in the scale of $r$, Scripta Math. 15 (1949), 11-12. MR 11:83g 
4. P. H. Cheo and Y. C. Yien, A problem on the $k$-adic representation of positive integers, Acta Math. Sinica 5 (1955), 433-438. MR 17:828b

5. H. Gupta, Selected Topics in Number Theory, ABACUS Press, 1980. MR 81e:10002

Department of Electrical and Computer Engineering, University of Florida, 435 Engineering Building, P.O. Box 116130, Gainesville, Florida 32611-6130

E-mail address: fang@ece.ufl.edu 